\title{
Formula Nano
}

\author{
Last April, six teams raced molecules on a metallic surface using a scanning tunnelling microscope in the first \\ NanoCar Race. The event brought scientific research in nanotechnology to the attention of the wider public.
}

Considering auto racing began five minutes after the second car was built - according to the famous Henry Ford quote - it took considerably longer for nanocars to engage in racing competition.

The idea of moving a molecule on a surface using an external input has been pursued with some degree of success for the past couple of decades. But important developments took place early this decade when a handful of novel designs were described in the literature ${ }^{1-4}$. This prompted Christian Joachim and Gwénaël Rapenne at the French National Center for Scientific Research (CNRS) in Toulouse and the University of Toulouse, respectively, to advance the idea that it would be fun and scientifically interesting to pit these nanocars against each other in an organized race ${ }^{5}$.

It took about four years for Joachim and his team to organize the first NanoCar Race ${ }^{6}$. They made available a unique scanning tunnelling microscope setup at CNRS that allows four independent tips to operate on different regions of a surface at the same time, wrote down the rules, found sponsors, promoted the event and organized training sessions in Toulouse for the teams involved. Finally, on 28 and 29 April 2017, six teams from around the world were ready to race, four on the track in Toulouse and two remotely (but controlled from Toulouse). The event, which Nature Nanotechnology helped sponsor, was significant for a number of reasons.

First, it was a genuine scientific project that fostered collaboration between chemists and physicists; broadly speaking, the former synthesizing the nanocars and the latter driving them. The NanoCar Race therefore exemplified the interdisciplinary character that has always been at the core of the nanotechnology endeavour.

Second, it rekindled broad interest among scientists for the field of atomic and molecular manipulation on a surface, which has seen ups and downs in the past two decades, but has never convincingly emerged to the fore. The NanoCar Race generated excitement and, hopefully, some impetus for developing better nanocars.

Third, it promoted scientific research to the wider public. The notion of a car race at the smallest possible scale is easy to grasp and to associate with more famous car races. The

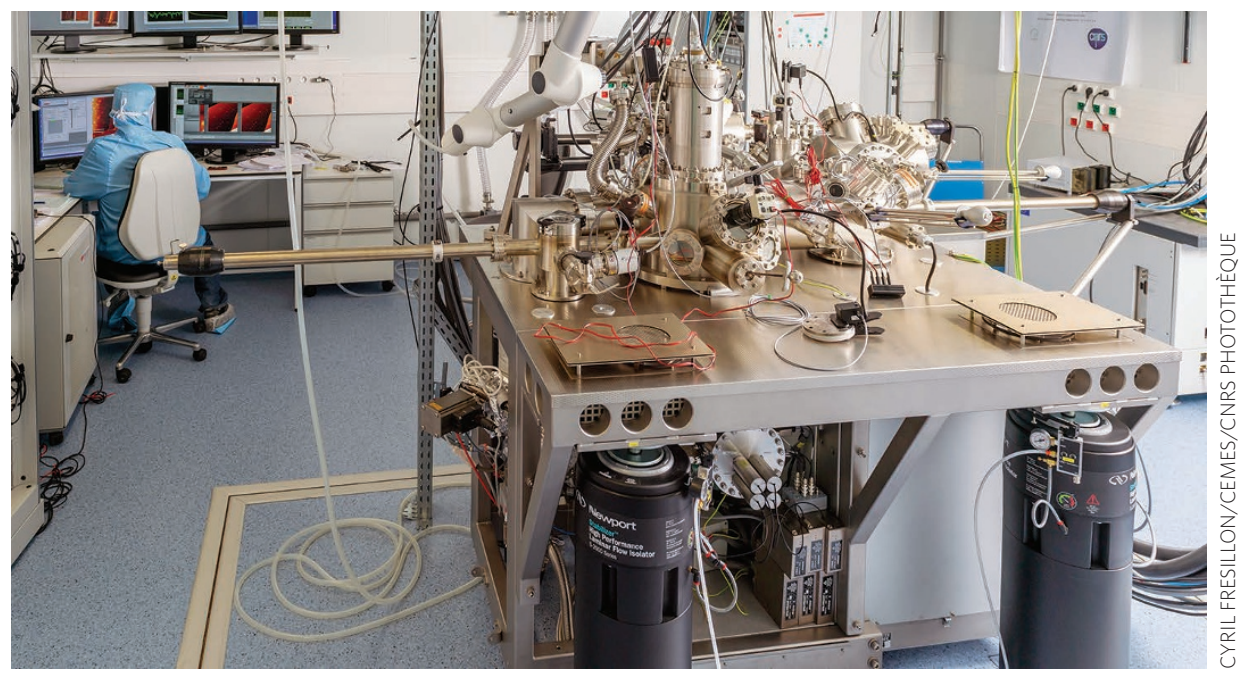

The four-tip scanning tunnelling microscope used for the NanoCar Race.

organizers cleverly capitalized on this and promoted the event with tutorial videos and live updates on their website. Moreover, the race was streamed in real time on YouTube. There, one could see scientists operate the scanning tunnelling microscope that powered their molecules. The NanoCar Race literally opened the doors of a world-class scientific laboratory to the wider public, showing scientists performing an experiment live.

For these reasons, we believe that the initiative should be commended. And in this issue, we host contributions by the two winners of the race - yes, there was an ex aequo.

Rémy Pawlak and Tobias Meier from the Swiss team from the University of Basel provide this month's In the Classroom column (p. 712), describing their experience from when they first heard about the NanoCar Race to race day itself. Their Swiss Nano Dragster went through various levels of troubleshooting before being ready for the event and crossing the finish line in 6 hours and 30 minutes. The experience of the Swiss team reinforces the fact that, beyond the fanfare of the general media, the NanoCar Race was the culmination of a genuine piece of scientific research.

In a Commentary (p. 604), the members of the American-Austrian team led by James Tour at Rice University and Leonard Grill at the University of Graz, share their advice on what makes a fast nanocar. Their Dipolar Racer was so fast, in fact, that it had to race on silver, rather than gold. Even with a penalty of an extra $50 \mathrm{~nm}$ to cover, they finished the race in 1 hour and 33 minutes. Overall, it travelled a micrometre in 29 hours, a world record. This kind of result would probably not have been possible without the American-Austrian team's long tradition of making and driving nanocars. We believe their advice will be valuable for other researchers in the field.

Although the first NanoCar Race was generally a success ${ }^{7}$, the event should be repeated in the future. We agree with the American-Austrian team that more stringent and challenging requirements should be implemented. Nanocars could be required to possess an electric motor unit, perform a specific task, or make an assembly of atoms, for example. This would further promote surface science and nanotechnology research, propelling it to excellence through (a fun) competition. Perhaps a Formula Nano is in the making. $\square$

\footnotetext{
References

1. Kudernac, T. et al. Nature 479, 208-211 (2011).

2. Chu, P.-L. E. et al. ACS Nano 7, 35-41 (2013).

3. Nickel, A. et al. ACS Nano 7, 191-197 (2013).

4. Perera, U. G. E. et al. Nat. Nanotech. 8, 46-51 (2013).

5. Joachim, C. \& Rapenne, G. ACS Nano 7, 11-14 (2013)

6. Castelvecchi, D. Nature 544, 278-279 (2017).

7. Rapenne, G. \& Joachim, C. Nat. Rev. Mater. 2, 17040 (2017).
} 